\title{
Vehicle-induced force on pedestrians
}

\author{
A. Sanz-Andrés \\ A. Laverón \\ A. Cuerva \\ C. Baker
}

\begin{abstract}
The main object of this study is to contribute to the study of the train-induced force on pedestrians with a theoretical model based on unsteady potential flow. The same method can be applied to other bodies and other kind of moving vehicles.

The outcome of this theoretical model is that the force coefficient (referred to the vehicle speed and the pedestrian cross-section diameter) acting on the pedestrian are proportional to a single parameter which involves the pedestrian cross-section diameter, the vehicle cross-section area and the distance between the pedestrian and the vehicle.

The results of the present model concerning the change in modulus and orientation experienced by the pedestrian, as the vehicles pass by, has a similar appearance to that considered in the European standards.

The results obtained are mainly qualitative because of the simplifying assumptions needed to obtain a simple formulation leading to analytical results, except in the case of a vehicle with streamlined front shapes, where quantitative results can be expected.
\end{abstract}

\section{Introduction}

High speed train operation creates many problems for railways companies, due to transient aerodynamic effects: lateral wind gust, pressure waves in tunnels, pressure applied to passing trains (unsteady aerodynamic effects associated with the passing 


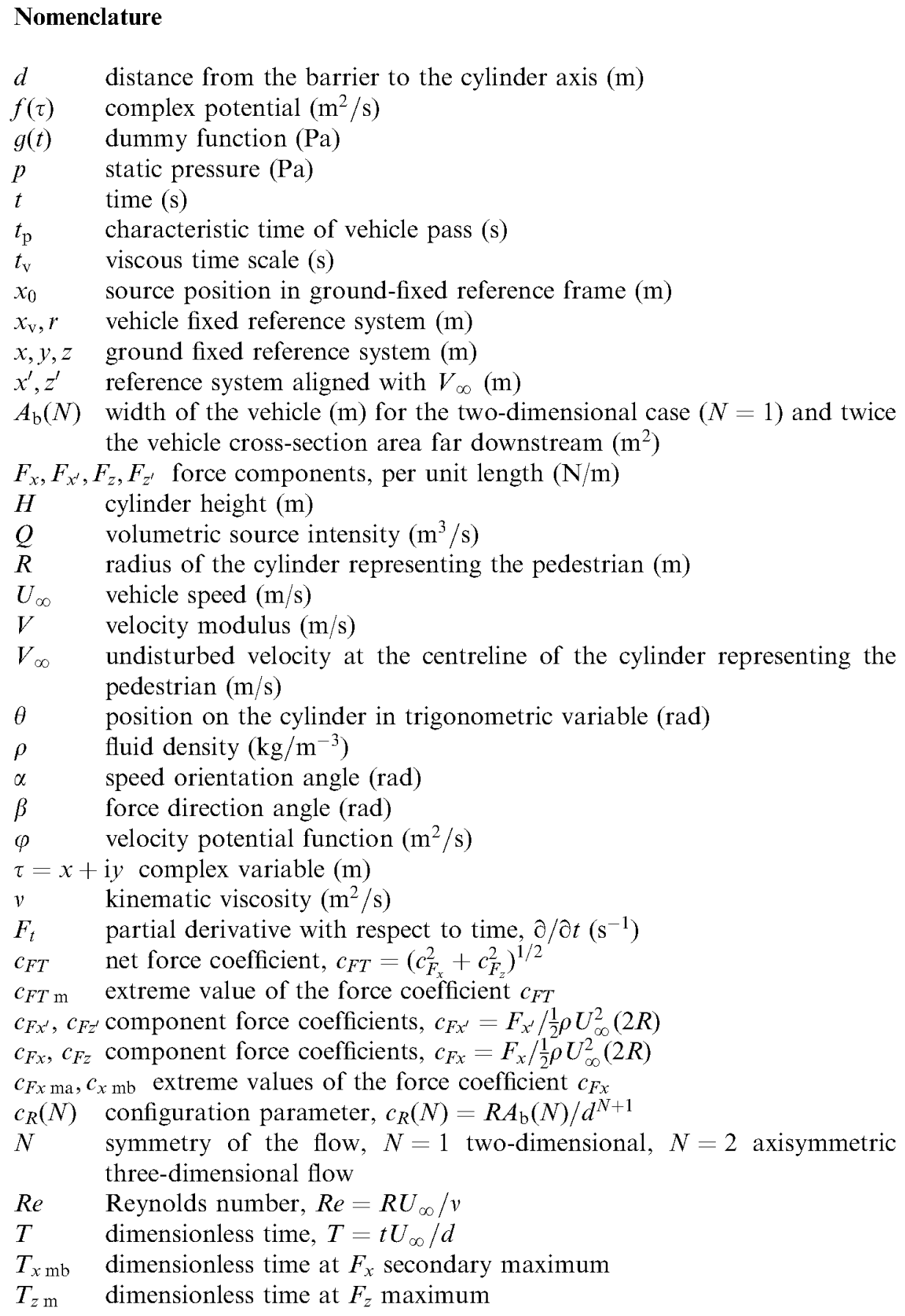


of the nose of high-speed trains and of the slipstreams and the tail wake, dynamic excitation, coupled with the aerodynamic forces generated by the train), etc.

One of the main problems that evidence the reality of the technical and economic impact is the safety of persons standing in the neighbourhood of the track, when placed under the effect of the vehicle-induced aerodynamic loads.

A basis for industrial development of appropriate solutions and widely accepted guidelines is needed in order to allow for a deeply founded development of the transport sector. In spite of the fact that this development is very important, there is a lack of published research in the field of unsteady loads induced on objects placed close to the tracks (pedestrians, structures, traffic signs, etc.) by the train when it passes.

The main object of this study is to contribute to the study of the train-induced load on pedestrians (although the same method can be applied to other bodies and other kind of moving vehicles). There are a few studies published concerning the pressure induced by a vehicle on pedestrians [1], vehicle-induced forces on flat plates and in traffic signs models [2], and full-scale measurements [3,4], which are mainly experimental. Some attempts have been made in order to explain, based on theoretical models, the observed time variation intensity of the load:

- in Ref. [5] the pressure variation with time measured in Ref. [1] was explained by using a simple mathematical model, although the comparison was limited due to the lack of available data concerning the values of the experimental parameters.

- in Refs. [6,7] the force variation (reported in Refs. [2,3]) measured on traffic signs was compared with a mathematical model, extension of the model presented in Ref. [5], and good qualitative (in some cases also quantitative) agreement was reported.

However, in the theoretical models presented in Refs. [5-7], the case of the force on a pedestrian was not considered, which is the aim of the present paper.

Following the method developed in Refs. [5-7], the aim of this paper is to obtain simple expressions for the speed generated by the vehicle motion and for the dependence with time of the force generated on the pedestrian, simple but able to explain the main characteristics of the phenomenon. The absence of appropriate experimental results published in the literature does not allow proper experimentalto-theoretical result's comparison. In spite of that, the validity of the model developed here is based on the comparison with the results of the theoretical models already developed for traffic signs. Additionally, the parallelism between the solutions of the two problems is established.

The model assumptions, as explained in Ref. [6], are based on the results of the full-scale measurements [3], which show "that the wind-induced forces can be treated separately from vehicle-induced loads, because the latter ones are generated largely by the transient pressure field induced by the vehicle motion relative to the sign and not by the motion of air".

The outcome of the mentioned theoretical models and experimental studies is that the main parameters that influence the force coefficient (referred to the vehicle speed 
and the sign area) acting on a sign are: the sign size, the sign orientation, the vehicle cross-section area, and the distance between the sign and the vehicle.

Of course, the nose shape is also important, but the study of this factor requires more complex mathematical (or numerical) models yet to be developed.

The action of the train on the pedestrian should be similar to that on a traffic sign: when the vehicle passes an unsteady pressure force appears attracting the sign towards the vehicle, phenomenon which can be traced back to a potential flow effect. The aim of the paper is to cover the absence of published theoretical models (mainly analytical) in which the test methods could be based upon, or even better, new experimental simplified methods could be designed.

The model developed here is an adaptation of the one presented in Refs. [5-7], which is based on the following assumptions:

(1) The flow generated by the vehicle motion is considered as a potential one. The wake effect is not considered here and the attention is focused on the vehicle nose effects.

(2) The potential flow is generated by a moving source, of intensity $Q$. Two cases are considered:

(a) a tall vehicle close to the pedestrian (two-dimensional flow around the vehicle);

(b) a not-so-tall vehicle or pedestrian not-so-close to the vehicle (threedimensional flow around the vehicle).

(3) The transversal size of the pedestrian is small compared to both the vehicle transversal size and the vehicle-to-pedestrian distance. Therefore, the influence of the pedestrian in the flow generated by the vehicle is small. The existence of two length scales allow us to say that the problem can be split into two simpler problems:

(a) a uniform (and steady in body-fixed reference frame) incident flow that flows around the vehicle (neglecting the pedestrian), which dominates the whole flow field, and

(b) a uniform but unsteady flow around the pedestrian, which is relevant only close to the pedestrian at distances of the order of the pedestrian diameter.

(4) The pressure field on the pedestrian is obtained by using the Bernoulli equation for unsteady flows.

(5) As shown in Ref. [7] no wake is developed behind the cylinder at the first moments of the vehicle pass, as the vehicle pass is very quick, thus the primary forces on the cylinder just at the first moments are a result of rapid acceleration of the fluid. As shown below, the wake is not developed at the first vehicle passage time interval. The characteristic time of vehicle pass is $t_{\mathrm{p}}=R / U_{\infty}$, where $R$ is the cylinder radius and $U_{\infty}$ the vehicle speed, larger than $10 \mathrm{~m} / \mathrm{s}$ in the cases of interest. The viscous time scale needed to develop a boundary layer leading to the production of a wake behind the cylinder, containing vortices, is of the order of $t_{\mathrm{v}}=R^{2} / v$, where $v$ is the kinematic viscosity. Therefore, $t_{\mathrm{p}} / t_{\mathrm{v}}=$ $v /\left(R U_{\infty}\right)=1 / R e$, where $R e$ is the Reynolds number which usually is $R e \gg 1$. Therefore, the pass is so quick that there is not time enough for a wake to be developed on the flow behind the cylinder. 
Following the above-mentioned scheme, the flow generated by a source will be first considered. Then the flow around the pedestrian body will be analysed. Finally the matching of both problems will give expressions for the force acting on the pedestrian.

\section{Flow generated by the vehicle motion}

The configuration considered is sketched in Fig. 1. In a reference frame $\left(x_{\mathrm{v}}, r\right)$ fixed to the vehicle, it is represented by a source placed in an incoming steady, uniform flow $U_{\infty}$, along the $x$-axis (the direction of the vehicle motion). Two different limiting situations can be considered. If the vehicle has a rectangular cross-section shape and the pedestrian is close to the tracks (in case of trains) or to the road (in case of road traffic) the flow induced by the vehicle at the pedestrian position is nearly two dimensional, so that the vehicle shape (in what concerns the flow close to the pedestrian) can be considered as generated by a vertical source line (a twodimensional source, that is, a source line parallel to $y$-axis, case denoted as $N=1$ ). If the pedestrian is far enough away, the flow produced by the vehicle at the pedestrian position can be considered as axisymmetric, and therefore to estimate the incoming flow impinging in the pedestrian the shape of the vehicle can be considered as generated by a three-dimensional source (denoted as $N=2$ ), as the case sketched in Fig. 1. The second case, one could consider the situation to be analogous to the far field behaviour of the inner field in the slender body problem, where the nonaxisymmetric flow field that appears close to a non-axisymmetric slender body becomes axisymmetric as the distance from the body increases [8].

Therefore, for a vehicle with a nose shape similar to the one generated by a two- or three-dimensional source the present model will give two limiting values of the force, the two-dimensional limit (valid if the pedestrian is close to the vehicle) and the three-dimensional limit, which applies at larger distances.

The velocity induced by a moving source, placed at $x_{0}=U_{\infty} t$ (the position of the source in ground-fixed reference frame), on the pedestrian position is given by Sanz Andrés et al. [6].

$$
V_{\infty}=\frac{Q}{2 \pi N} \frac{1}{\left[x_{0}^{2}+d^{2}\right]^{N / 2}},
$$

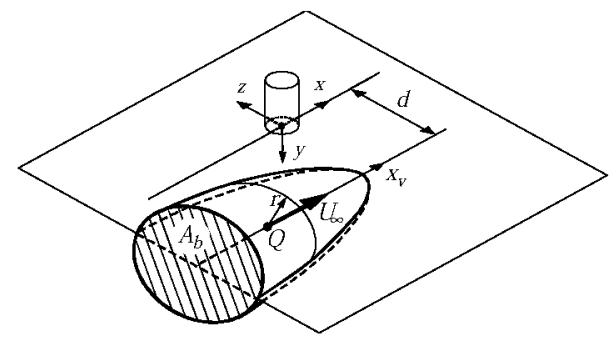

Fig. 1. Sketch of the configuration considered. $\left(r, x_{\mathrm{v}}\right)$ vehicle fixed reference system. $(x, y, z)$ ground fixed reference system. $d$, distance from the vehicle path to the pedestrian location. $Q$, source intensity. $A_{\mathrm{b}}$, cross-section area. The axisymmetric source case $(3 \mathrm{D}, N=2)$ is represented. 
where $N=1,2$ denotes two- (2D) or three-dimensional (3D) flow fields, respectively. $d$ is the distance from the pedestrian to the moving vehicle middle plane. $U_{\infty}$ is the vehicle motion speed. The time origin $t=0$ represents the closest position of the source with regard to the pedestrian and, from now on, it is named as "vehicle pass origin".

The orientation angle of the flow impinging in the pedestrian, $\alpha$, is given by

$$
\sin \alpha=\frac{d}{\left[x_{0}^{2}+d^{2}\right]^{1 / 2}}
$$

or

$$
\alpha=\tan ^{-1}\left(\frac{d}{-x_{0}}\right) .
$$

The source intensity, $Q$, is determined based on mass flow considerations to be

$$
Q=U_{\infty} A_{\mathrm{b}}(N)
$$

where $A_{\mathrm{b}}(N)$ is the moving body cross-section area far downstream, where the incident flow speed $U_{\infty}$ (in vehicle-fixed reference frame) is recovered. The vehicle itself and its image with regard to the ground plane form the moving body. In a twodimensional configuration $A_{\mathrm{b}}(\mathrm{m})$ represents the area of the cross-section per unit length, that is, the vehicle width. $A_{\mathrm{b}}\left(\mathrm{m}^{2}\right)$ represents the area of the vehicle far downstream for the three-dimensional case.

The shape of the bodies obtained in such a way is rather streamlined, but a more representative shape could be obtained by using a superposition of sources, sinks and doublets, although at this stage just a rough model of the vehicle is enough for the aimed purposes.

\section{Flow around the pedestrian}

The pedestrian can be represented as a circular cross-section cylinder of radius $R$ and height $H$, as shown in Figs. 1 and 2. The flow generated by the source that impinges in the pedestrian has a magnitude $V_{\infty}\left(x_{0}\right)$ and direction $\alpha\left(x_{0}\right)$, which depends on the position of the source with regard to the pedestrian position (Fig. 2). $V_{\infty}$ is the undisturbed velocity at the centreline of the cylinder representing the pedestrian.

Close to the pedestrian, if $H \gg R$, around each $y$ section (except close to the cylinder ends) an almost two-dimensional flow is established.

The solution of the two-dimensional problem is obtained by using the complex potential formulation, $f(\tau)$, where $\tau=x+\mathrm{i} z$ is the complex variable,

$$
f(\tau)=V_{\infty}\left(\mathrm{e}^{-\mathrm{i} \alpha} \tau+\mathrm{e}^{\mathrm{i} \alpha} R^{2} / \tau\right) .
$$

The velocity potential function $\varphi$ on the cylinder surface $\left(\tau=R \mathrm{e}^{\mathrm{i} \theta}\right)$ is given by

$$
\varphi=\operatorname{Re}[f(\tau)]=2 V_{\infty} R \cos (\theta-\alpha),
$$




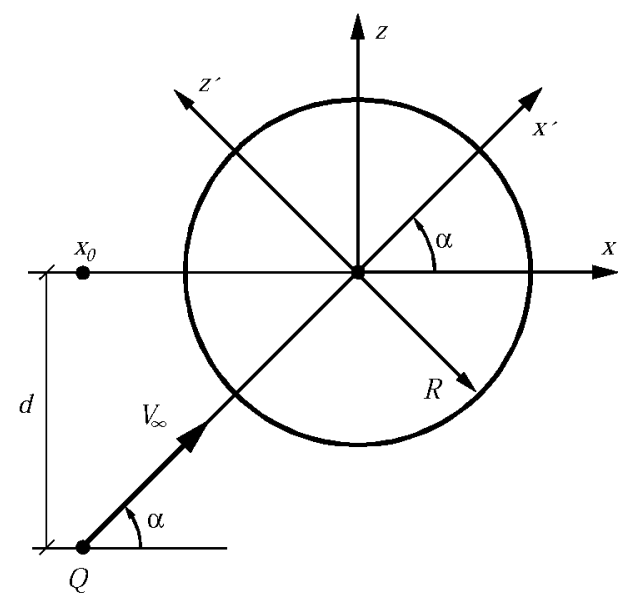

Fig. 2. Model of the flow generated by the vehicle impinging the pedestrian. $V_{\infty}$, flow speed at the pedestrian position generated by the source $Q . \alpha$, flow direction. $(x, z)$, ground fixed reference frame. $\left(x^{\prime}, z^{\prime}\right)$, rotating reference frame oriented along incoming flow direction. $d$, distance from the vehicle path to the pedestrian location.

where Re denotes the real part. Expression (6) is the classical solution for the potential flow of a uniform incident stream around a circular cylinder, but rotated an angle $\alpha$.

The pressure field is obtained by using the Bernoulli equation for a non-steady potential flow field

$$
p+\frac{1}{2} \rho V^{2}+\rho \varphi_{t}=g(t)
$$

where $p, V$ and $\rho$ are the static pressure, the velocity modulus and the fluid density, respectively, of the flow around the cylinder representing the pedestrian. $g(t)$ is a function of time.

In the calculation of the force acting on the cylinder the dynamic pressure term $\left(\frac{1}{2}\right) \rho V^{2}$ will not contribute (due to the symmetry of the velocity field) and the $g(t)$ term is uniform on the whole flow field, so that its integral contribution to the force on the cylinder will be zero. Taking into account the only one term contributing to the force, $\rho \varphi_{t}$, the pressure on the cylinder surface is reduced to

$$
p=-\rho \varphi_{t}=-2 \rho R\left[\dot{V}_{\infty} \cos (\theta-\alpha)+\dot{\alpha} V_{\infty} \sin (\theta-\alpha)\right] .
$$

The force components $F_{x}$ and $F_{z}$ are given by

$$
\begin{aligned}
& F_{x}=-\int_{0}^{2 \pi} p(\theta) \cos \theta R \mathrm{~d} \theta=2 \pi \rho R^{2}\left[\dot{V}_{\infty} \cos \alpha-\dot{\alpha} V_{\infty} \sin \alpha\right], \\
& F_{z}=-\int_{0}^{2 \pi} p(\theta) \sin \theta R \mathrm{~d} \theta=2 \pi \rho R^{2}\left[\dot{V}_{\infty} \sin \alpha+\dot{\alpha} V_{\infty} \cos \alpha\right] .
\end{aligned}
$$


These results can be considered as if the force would be a combination of two simpler force contribution $F_{x^{\prime}}$ and $F_{z^{\prime}}$ along axes $x^{\prime}$ and $z^{\prime}$ ( $x^{\prime}$ directed along $V_{\infty}$ direction, and $z^{\prime}$ normal to $x^{\prime}$ ), as shown in Fig. 2, given by

$$
\begin{aligned}
& F_{x^{\prime}}=2 \pi \rho R^{2} \dot{V}_{\infty}, \\
& F_{z^{\prime}}=2 \pi \rho R^{2} \dot{\alpha} V_{\infty} .
\end{aligned}
$$

The force component $F_{x^{\prime}}$ is generated by the acceleration of the fluid along $x^{\prime}$, and the force component $F_{z^{\prime}}$ is due to the change of direction of the impinging flow.

The force coefficients in dimensionless form are given by

$$
\begin{gathered}
c_{F x^{\prime}}=\frac{F_{x^{\prime}}}{\frac{1}{2} \rho U_{\infty}^{2}(2 R)}=2 \pi R \frac{\dot{V}_{\infty}}{U_{\infty}^{2}}, \\
c_{F z^{\prime}}=\frac{F_{z^{\prime}}}{\frac{1}{2} \rho U_{\infty}^{2}(2 R)}=2 \pi R \frac{\dot{\alpha} \boldsymbol{V}_{\infty}}{U_{\infty}^{2}} .
\end{gathered}
$$

Substituting $V_{\infty}, \dot{V}_{\infty}$ and $\dot{\alpha}$ from Eqs. (1) and (3) and using the dimensionless time $T$

$$
T=t \frac{U_{\infty}}{d},
$$

expressions (13) and (14) become

$$
\begin{aligned}
& c_{F x^{\prime}}=c_{R}(N) \frac{-T}{\left(T^{2}+1\right)^{N / 2+1}}, \\
& c_{F z^{\prime}}=\frac{c_{R}(N)}{N} \frac{1}{\left(T^{2}+1\right)^{N / 2+1}},
\end{aligned}
$$

where

$$
c_{R}(N)=\frac{R A_{\mathrm{b}}(N)}{d^{N+1}}
$$

is the configuration parameter. This parameter shows the dependence of the force coefficient on the several geometrical parameters involved: the pedestrian crosssection radius, the vehicle cross-section area and the distance between the pedestrian and the vehicle.

The force component coefficients referred to axes $x$ and $z$ are given by

$$
\begin{aligned}
& c_{F x}=c_{F x^{\prime}} \cos \alpha-c_{F z^{\prime}} \sin \alpha=\frac{c_{R}}{N} \frac{N T^{2}-1}{\left(T^{2}+1\right)^{(N+3) / 2}}, \\
& c_{F z}=c_{F x^{\prime}} \sin \alpha+c_{F z^{\prime}} \cos \alpha=c_{R} \frac{1+N}{N} \frac{-T}{\left(T^{2}+1\right)^{(N+3) / 2}} .
\end{aligned}
$$

The variation with time $T$ of the force component coefficients $c_{F x}$ and $c_{F z}$ are plotted in Fig. 3. 


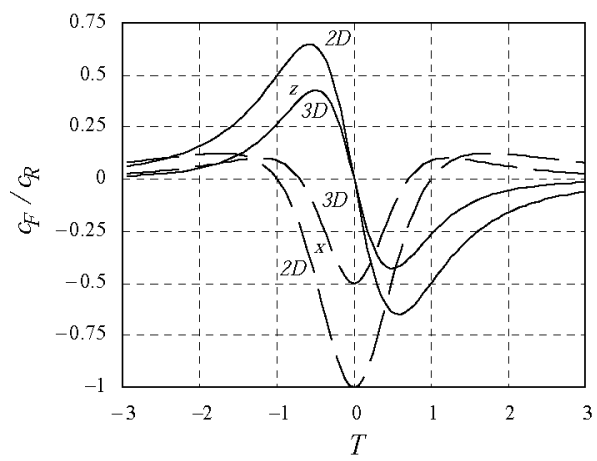

Fig. 3. Variation of the force coefficient, $c_{F}$, with the dimensionless time, $T . c_{R}$ is the configuration parameter. 2D (3D), two- (three-) dimensional source. Solid line (symbol z), transversal force coefficient $c_{F z}$. Dashed line (symbol $x$ ) longitudinal force coefficient $c_{F x} . c_{F x}>0$ means force pushing in the vehicle direction. $c_{F Z}>0$ means force pushing out of the road.

Concerning the longitudinal force (along track), $c_{F x}$, the force expression (19) is very similar to the force generated by a passing vehicle on a traffic sign placed normal to the track [6]: first a small force pushes in the vehicle direction when the vehicle is approaching, but a strong peak in the opposite direction to the vehicle motion occurs when the vehicle leading edge is close to pass the sign [2]. The time evolution is symmetric with regard to the vehicle pass origin (the pass of the source in front of the pedestrian). The extreme values of the force, $c_{F x}$ ma, occurs at $T=0$, the vehicle pass origin

$$
c_{F x \mathrm{ma}}=-\frac{c_{R}(N)}{N}=-\frac{R A_{\mathrm{b}}(N)}{N d^{N+1}},
$$

which gives a first estimation of the influences of the parameters $A_{\mathrm{b}}, d, R$ and $N$.

The secondary maximum occurs at $T=T_{x \mathrm{mb}}$

$$
T_{x \mathrm{mb}}= \pm \sqrt{\frac{3}{N}}
$$

and the associated relative maximum value is $c_{F x} \mathrm{mb}$

$$
c_{F x \mathrm{mb}}=c_{R} \frac{2}{N}\left(\frac{N}{N+3}\right)^{(N+3) / 2}=\frac{R A_{\mathrm{b}}(N)}{d^{N+1}} \frac{2}{N}\left(\frac{N}{N+3}\right)^{(N+3) / 2},
$$

where

$$
\begin{aligned}
& c_{F x \mathrm{mb}}(1) \cong 0.125 \frac{R A_{\mathrm{b}}(1)}{d^{2}}, \\
& c_{F x \mathrm{mb}}(2) \cong 0.101 \frac{R A_{\mathrm{b}}(2)}{d^{3}} .
\end{aligned}
$$


The relation between extreme values is

$$
\begin{aligned}
& \left|\frac{c_{F x \mathrm{mb}}}{c_{F x \mathrm{ma}}}\right|=2\left(\frac{N}{N+3}\right)^{(N+3) / 2}=0.125 \text { for } N=1, \\
& \left|\frac{c_{F x \mathrm{mb}}}{c_{F x \mathrm{ma}}}\right|=0.202 \text { for } N=2
\end{aligned}
$$

and can be used, together with the position of the extreme values, to characterize the experimental results.

Concerning the transversal force coefficient $c_{F z}$, given by Eq. (20), the result is very similar to the force experimented by a traffic sign placed parallel to the vehicle motion (Fig. 3): the sign is pushed out of the road when the vehicle is approaching $(T<0)$ (which is also the phenomenon experienced when a train crosses with another running in the opposite direction in a parallel track) and it is pulled towards the vehicle when the leading edge of the vehicle passes the sign. The time evolution is antisymmetric with regard to the vehicle pass origin $(T=0)$.

The extreme values of the transversal force coefficient, $c_{F z}$, occurs at $T=T_{z \mathrm{~m}}$,

$$
\begin{aligned}
& T_{z \mathrm{~m}}= \pm \sqrt{\frac{1}{N+2}} \\
& \left|c_{F z} \mathrm{~m}\right|=c_{R}(N) c_{z \mathrm{~m}}(N)=\frac{R A_{\mathrm{b}}(N)}{d^{N+1}}\left(\frac{N+1}{N}\right) \frac{(N+2)^{(N+2) / 3}}{(N+3)^{(N+3) / 2}}
\end{aligned}
$$

where

$$
\begin{aligned}
& c_{F z \mathrm{~m}}(N)=\frac{N+1}{N} \frac{(N+2)^{(N+2) / 2}}{(N+3)^{(N+3) / 2}}, \\
& c_{F z \mathrm{~m}}(1) \cong 0.650 \frac{R A_{\mathrm{b}}(1)}{d^{2}}, \\
& c_{F z \mathrm{~m}}(2) \cong 0.429 \frac{R A_{\mathrm{b}}(2)}{d^{3}} .
\end{aligned}
$$

As in the previous case, expression (29) gives the influence of the configuration parameters included in $c_{R}(N)$ and of the flow characteristic (through $c_{z} \mathrm{~m}(N)$ ).

The component force coefficients, $c_{F x}$ and $c_{F z}$ on a cylinder have twice the value of the force coefficient on a sign (flat plate model), oriented normal and parallel to the road, respectively, found in Ref. [6].

Eqs. (19) and (20) can be combined to obtain the net force coefficient, $c_{F T}$, and the force direction angle $\beta$, defined as follows:

$$
\begin{aligned}
c_{F T}=\left(c_{F x}^{2}+c_{F z}^{2}\right)^{1 / 2} & =c_{R}(N) \frac{1}{N} \frac{\left[N^{2} T^{4}+\left(1+N^{2}\right) T^{2}+1\right]^{1 / 2}}{\left(T^{2}+1\right)^{(N+3) / 2}} \\
& =\frac{R A_{\mathrm{b}}(N)}{d^{N+1}} \frac{\left[N^{2} T^{4}+\left(1+N^{2}\right) T^{2}+1\right]}{N\left(T^{2}+1\right)^{(N+3) / 2}},
\end{aligned}
$$




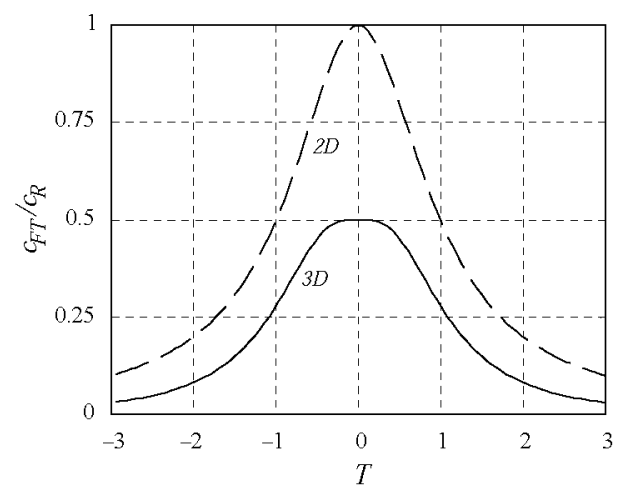

Fig. 4. Variation of the net force coefficient, $c_{F T}$, with the dimensionless time, $T . c_{R}$ is the configuration parameter. 2D (3D) two- (three-) dimensional source.

$$
\tan \beta(T, N)=\frac{c_{F z}}{c_{F x}}=(1+N) \frac{-T}{N T^{2}-1}=\frac{1+N}{2} \frac{\sin 2 \alpha}{N \cos ^{2} \alpha-\sin ^{2} \alpha} .
$$

The net force coefficient evolution with time, $c_{F T}(T)$, is shown in Fig. 4 for $2 \mathrm{D}$ and $3 \mathrm{D}$ flows, where the symmetry with regard to vehicle pass origin $T=0$ can be appreciated. The maximum value of the force coefficient $c_{F T} \mathrm{~m}$ occurs at vehicle pass origin $T=0$ and is given by

$$
c_{F T \mathrm{~m}}=\frac{c_{R}(N)}{N}=\frac{R A_{\mathrm{b}}(N)}{N d^{N+1}}, \quad c_{F T \mathrm{~m}}(1)=\frac{R A_{\mathrm{b}}(1)}{d^{2}}, \quad c_{F T \mathrm{~m}}(2)=\frac{R A_{\mathrm{b}}(2)}{2 d^{3}},
$$

where $N$ shows the influence on the force of the flow characteristics $(2 \mathrm{D}$ and $3 \mathrm{D}$ flows).

The force direction evolution with time, $\beta(T, N)$, given by Eq. (34), is shown in Fig. 5a, where the influence of the dimensionality of the flow, and the sudden direction change in the range $-1<T<1$ can be appreciated. In particular, it can be shown from Eq. (34), that in the $2 \mathrm{D}$ case $(N=1)$ the force orientation angle is

$$
\beta(T, 1)=2 \alpha(T) .
$$

The variation of the force direction $\beta$, with the incoming flow direction $\alpha$, is plotted in Fig. 5b. In the 3D case the evolution $\beta(\alpha)$ is close to the $2 \mathrm{D}$ case.

The change of force direction is as follows. As $\alpha(T)$ increases from 0 to $\pi$ then $\beta(T)$ changes from 0 to $2 \pi$, that is, the force direction changes monotonously with time. At the beginning $(\alpha=0)$ the force is oriented towards the vehicle motion direction ( $\beta=0$ ); when the vehicle is passing $(\alpha=\pi / 2)$ the force acts in the direction opposite to the vehicle motion $(\beta=\pi)$; when $\alpha=3 \pi / 4$ the force pushes the pedestrian towards the track $(\beta=3 \pi / 2)$, and afterwards $(\alpha=\pi)$ the force is oriented again in the vehicle direction $(\beta=2 \pi)$.

The maximum transversal force towards the vehicle occurs when $T=T_{z \mathrm{~m}}=$ $\sqrt{1 /(N+2)}$ and is given by Eq. (29) and is shown in Fig. 3. 

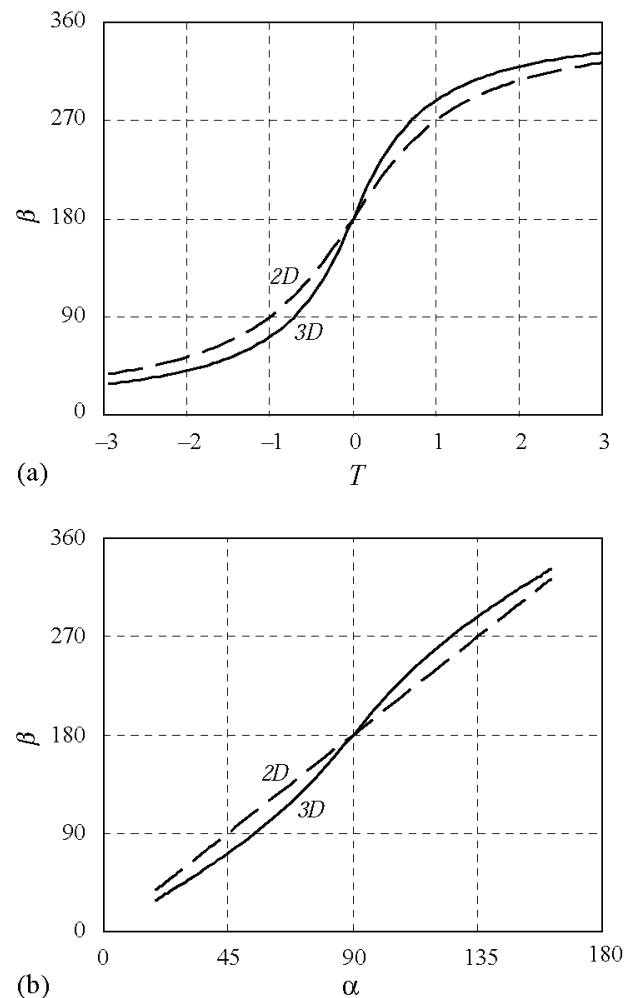

Fig. 5. (a) Variation of the force direction $\beta$ with the dimensionless time, T. 2D (3D) two- (three-) dimensional source. (b) Variation of the force direction, $\beta$, with the incoming flow direction angle, $\alpha, 2 \mathrm{D}$ (3D) two- (three-) dimensional source.

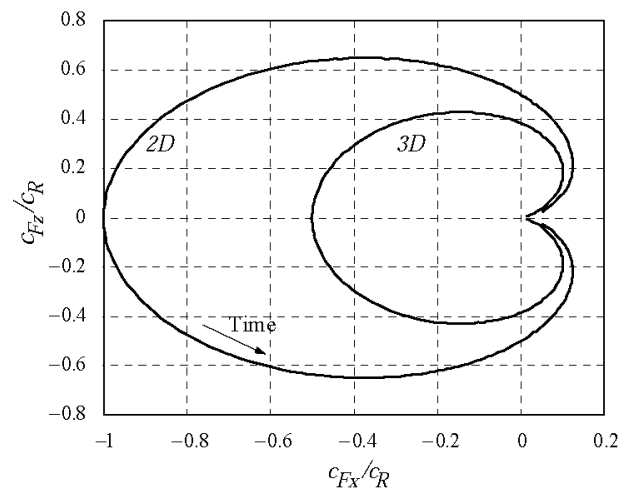

Fig. 6. Instantaneous force coefficient, $c_{F x}$ and $c_{F z}$ for a circular cylinder at the road on track side. $c_{R}$ is the configuration parameter. 2D (3D), two- (three-) dimensional source. $c_{F x}>0$ means force pushing in the vehicle direction. $c_{F Z}>0$ means force pushing out of the road. 
The variation of $c_{F z}$ with $c_{F x}$ is shown in Fig. 6. The change in modulus and orientation experienced by the pedestrian as the vehicles pass by can be clearly seen. This plot has a similar appearance to that of Fig. 7 in Ref. [9].

\section{Conclusions}

In this paper, the force acting on a pedestrian produced by a vehicle passing close to it has been modelized by using unsteady potential flow theory. The following conclusions can be outlined. The evolution of the total force as well as the force component parallel to the vehicle motion is symmetric with regard to the time origin (vehicle pass origin), and the maximum values appear at just this time origin.

On the other hand, the transversal force component, acting towards the vehicle path, is antisymmetric with regard to the vehicle pass origin, and the maximum value appears when the source representing the vehicle is at a distance

$$
d T_{z \mathrm{~m}}=d \sqrt{\frac{1}{N+2}}
$$

from the vehicle pass origin. The direction of the force changes during the vehicle pass, the angle being nearly twice the angle between the vehicle trajectory and the source pedestrian line. A sudden change in force direction happens when the vehicle is passing closest to the pedestrian, at distances in the range $-d$ to $d$ measured from the vehicle pass origin.

The force coefficient depends on the geometrical parameters involved (pedestrian cross-section radius, $R$, vehicle cross-section area $A_{\mathrm{b}}$, distance between them, $d$ ) through a non-dimensionless combination called configuration parameter:

$$
c_{R}(N)=\frac{R A_{\mathrm{b}}}{d^{N+1}} .
$$

The results obtained in this paper help to appraise an impression of the effect of a moving vehicle (train, trunk, etc.) on a body with volume (pedestrian, worker, masts, etc.) close to the vehicle path and the influence of the parameters involved. A curious result is that the force coefficient depends on both the diameter of the body and the cross-section area of the moving vehicle. Therefore, care should be taken when designing aerodynamic tests at reduced scale.

In the case of a pedestrian, taken as a dynamical system, the total effect depends on the applied transient force (described here) and the pedestrian response to this force. The problems for a pedestrian to keep in equilibrium during a vehicle pass could come from the intensity of the force (which can overpass the pedestrian available reaction force) or from the sudden and unexpected change in force direction (in such a way that the time constant of the pedestrian's nervous control system is too slow to catch up with the change, leading to control instability and loss of equilibrium). 
In the case of structures with volume (similar to the case of a pedestrian), the effect of the vehicle pass is to produce transient loads that can promote fatigue loads and failure of the structure.

The results obtained are mainly qualitative. The simplifying assumptions needed to obtain a simple formulation leading to analytical results are responsible for this quantitative character. It is clearly demonstrated by experiments [2] that the actual forces on overheads signs and, by inference, on pedestrians are a strong function of the shape of the front of the vehicle. However, according to Ref. [6] the simple model presented would produce results applicable to vehicle with streamlined front shapes.

\section{Acknowledgements}

The authors would like to thank Profs. J. Meseguer and J.M. Perales for helpful discussions, and recognize the contribution of the European Programme COST 14.

\section{References}

[1] H.J. Gerhardt, O. Krüger, Wind and train driven air movements in train stations, J. Wind Eng. Ind. Aerodyn. 74-76 (1998) 589-597.

[2] P.M. Cali, E.E. Covert, Experimental measurements of loads on an overhead highway sign structure by vehicle-induced gusts, J. Wind Eng. Ind. Aerodyn. 84 (2000) 87-100.

[3] A.D. Quinn, C.J. Baker, N.G. Wright, Wind and vehicle induced forces on flat plates. Part 2: vehicle induced force, J. Wind Eng. Ind. Aerodyn. 89 (2001) 831-847.

[4] K.W. Johns, R.J. Dexter, The development of fatigue design load ranges for cantilevered sign and signal support structures, J. Wind Eng. Ind. Aerodyn. 77\&78 (1998) 315-326.

[5] A. Sanz-Andrés, J. Santiago-Prowald, Train-induced pressure on pedestrians, J. Wind Eng. Ind. Aerodyn. 90 (2002) 1007-1015.

[6] A. Sanz-Andrés, J. Santiago-Prowald, C.J. Baker, A.D. Quinn, Vehicle induced loads on traffic sign panels, J. Wind Eng. Ind. Aerodyn. 91 (2003) 925-942.

[7] A. Sanz-Andrés, A. Laverón, C.J. Baker, A.D. Quinn, Vehicle induced loads on pedestrian barriers, J. Wind Eng. Ind. Aerodyn. (in review process).

[8] H. Ashley, M. Landahl, Aerodynamics of Wings and Bodies, Dover, New York, 1985, p. 112.

[9] European Standard, pr EN 14067-2, 2002, final draft. 